\title{
PROPERTIES OF DECAMETER SPIKES
}

\author{
V. N. Melnik*, N. V. Shevchuk*, H. O. Rucker ${ }^{\dagger}$, A. A. Konovalenko*, \\ V. V. Dorovskyy*, E. P. Abranin*, and A. Lecacheux ${ }^{\ddagger}$
}

\begin{abstract}
In this paper properties of decameter spikes observed in July - August, 2002 by radio telescope UTR-2 are discussed. These bursts have short duration (about 1s) and narrow frequency bandwidth $(50-70 \mathrm{kHz})$. They are situated chaotically on the dynamic spectrum at the decameter wavelengths. These bursts are weak, their fluxes are not higher than 200 - 300 s.f.u. One of the interesting features of decameter spikes is linear dependence of frequency bandwidth on frequency. Such dependence can be explained in the frame of plasma mechanism of radio emission if to take into account that Langmuir waves are generated into the angle about $20 \mathrm{deg}$ along the direction of electron beam propagation. In the paper the cause of small duration of spikes and generation of plasma waves in limited spatial regions are discussed.
\end{abstract}

\section{Introduction}

Elgaroy and de Groot [Malville et al., 1962] were the firsts who classified spike bursts as individual type of radio bursts. These bursts have short durations $(\tau<0.1 \mathrm{~s})$ and narrow bandwidths $(1-3 \%$ from the observational frequency). They are observed in a wide range from decimeter to meter wavelengths [Benz, 1986]. Spikes are often associated with different types of bursts such as type I bursts, type III bursts and type IV bursts. They are met in groups of bursts and sometimes they are a background radio emission for other bursts. Spikes have different frequency drift rates. It varies from zero drift rates to rates closer to frequency drift rates of Type III bursts [Tarnstrom et al., 1972]. There are different points of view on possible mechanism of spike generation [Tarnstrom et al., 1972; Malville, 1967; Zheleznyakov, 1975]. The fact that spikes occur simultaneously with type IV bursts indicates on the electron cyclotron maser mechanism of radiation. But most of authors [Tarnstrom et al., 1972; Zaitsev, 1974] are inclined to plasma mechanism. In

\footnotetext{
*Institute of Radio Astronomy, Ukrainian Academy of Sciences, Chervonopraporna 4, 61002, Kharkiv, Ukraine

† Space Research Institute, Austrian Academy of Sciences, Schmiedlstrasse 6, A-8042 Graz, Austria

‡LESIA, Observatoire de Paris, UMR CNRS 8109, 92195 Meudon, France
} 
favor of that says spikes associations with type I bursts and type III bursts.

In the present paper we analyze the observational properties of spikes at frequencies 10 - 30 MHz. It is turned out that decameter spikes are observed often and they have properties similar to those for spikes observed at higher frequencies.

\section{Observations}

For our analysis we used solar data obtained with the help of radio telescope UTR-2 and digital spectral polarimeter (DSP) [Kleewein, 1997]. This allowed to observe spikes in the wide frequency band $18-30 \mathrm{MHz}$ with the frequency resolution $12 \mathrm{kHz}$ and the time resolution 20,50 or $100 \mathrm{~ms}$. For the analysis we choose spikes which were observed during passage of the active region No.260 (Fig.1) across the solar disk from July 27 to August $2,2002$.

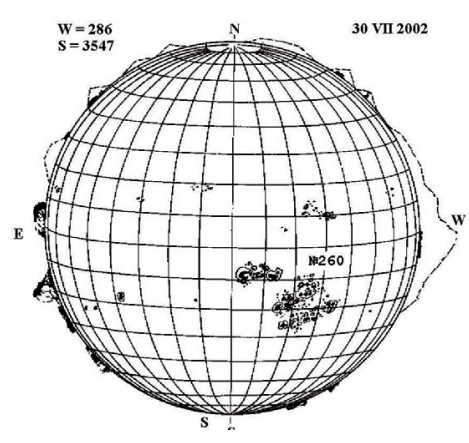

Figure 1: Location of active region No.260 on the solar disk on July 30.

On some days we observed the storms of such bursts. In these days there were some thousands of spikes chaotically placed on the dynamical spectrum (Fig.2). We measured the parameters of the bursts, which were observed during arbitrarily chosen 10 minutes interval for each day. There were about 350 spikes in every interval. More than 2000 of spikes for July 27 - August 2 were analyzed.

It is interesting to note that durations and instantaneous frequency bandwidths of spikes vary in fully determined ranges, the durations varies near value $1.2 \mathrm{~s}$ and the bandwidths varies near $50-70 \mathrm{kHz}$. Decameter spikes were observed simultaneously with type III bursts and type IV bursts. In this decameter spikes are similar to high frequency spikes. The frequency and time profiles of spike are practically symmetrical. The latter reminds the property of type III bursts, which also have similar time profile. But of course the duration of type III bursts is sometimes larger than that of spikes. This is another similarity with high frequency spikes. The small duration, narrow frequency bandwidth and chaotic appearance of spikes on dynamical spectrum distinguish them from other decameter bursts in individual type of solar bursts. 


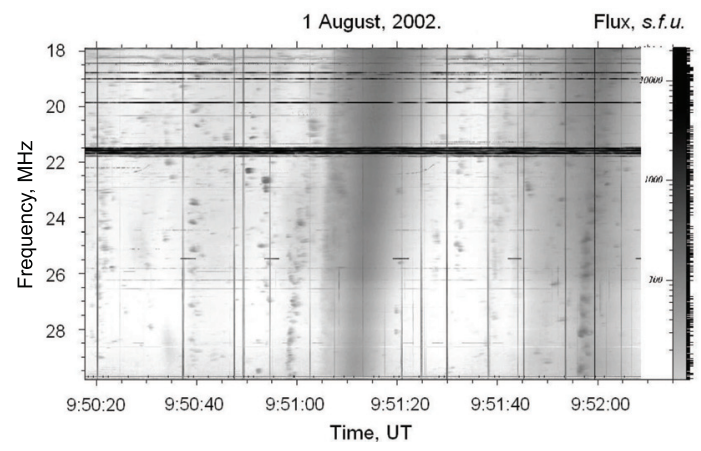

Figure 2: Decameter spikes against Type III bursts storm.

\subsection{Spike Durations}

Histograms for durations of spikes observed on August 1, 2002 on frequencies 19, 26 $\mathrm{MHz}$ are presented in Fig.3. Widths of these distributions are not very large and these distributions have obvious maximums.

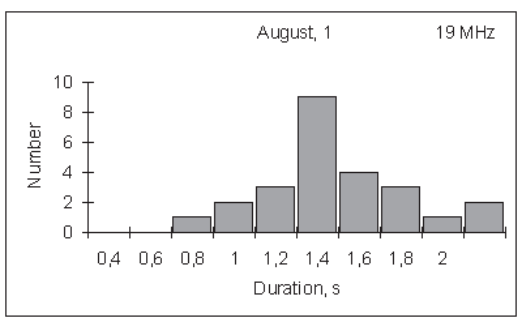

a)

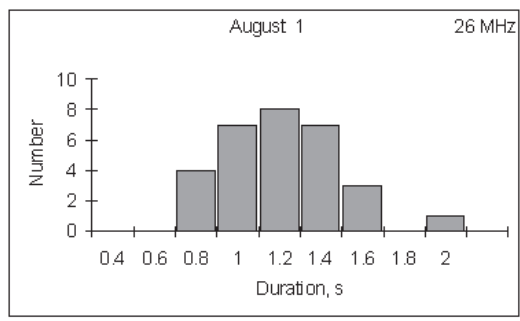

b)

Figure 3: The distribution of decameter spikes on their durations for August 1, 2002.

The minimum value of durations obtained is equal to $0.4 \mathrm{~s}$ and the maximum one is more than $2 \mathrm{~s}$. As we can see the distributions shift in the region of small durations with frequency.

From analysis of duration dependencies on frequency (Fig.4) it follows that they are in good agreement with the collision time dependency on frequency for plasma with temperature $10^{6} \mathrm{~K}$ (note that the collision time dependence for plasma with temperature $2 \cdot 10^{6} \mathrm{~K}$ lies essentially higher).

These dependencies practically concide at frequencies higher than $23-24 \mathrm{MHz}$ for all analyzed days. It is necessary to note that similar situation occurs for meter and decimeter spikes. Their durations are defined by collision time for plasma with temperature $10^{6} \mathrm{~K}$ too. Vicinity of decameter spike duration and collision time allows to define temperature of coronal plasma at heights $1-3 R_{s}$ ( $R_{s}$ is the solar radius) from observations of spikes at frequencies $10-30 \mathrm{MHz}$. 


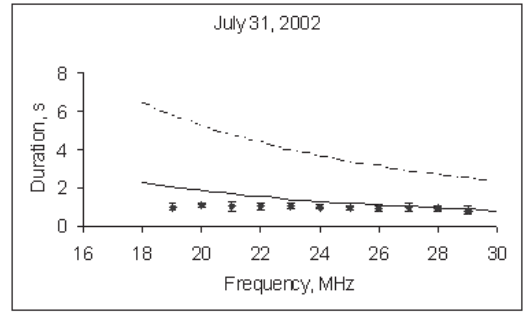

a)

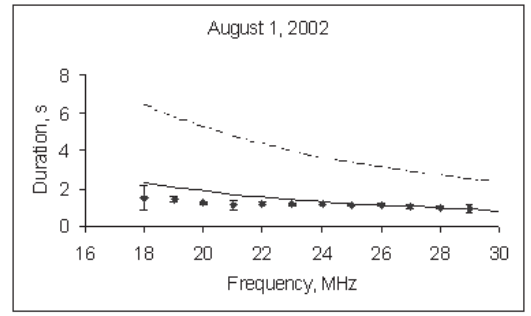

b)

Figure 4: The duration dependencies on frequency for July 31(a) and August 1(b), 2002. The solid line corresponds to plasma with temperature $10^{6} \mathrm{~K}$ and pointed line to plasma with temperature $2 \cdot 10^{6} \mathrm{~K}$.

\section{$2.2 \quad$ Frequency Bandwidths of Spikes}

The frequency profiles of spikes are symmetrical. The distributions of the bursts are rather compact (Fig.5) and bursts are grouped near average values even when the number of the bursts is not very large.

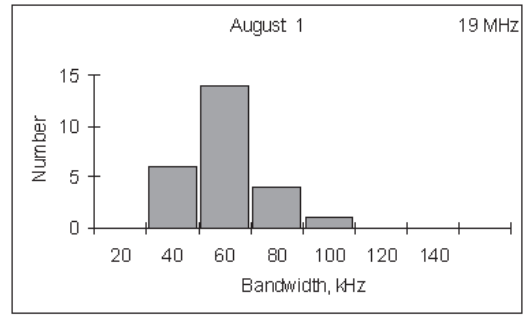

a)

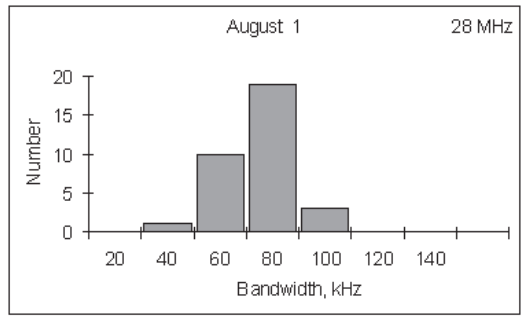

b)

Figure 5: The distribution of decameter spikes on frequency bandwidth for August 1, 2002.

The average values of bandwidths on different frequencies are increase with frequency. The dependencies of bandwidth on frequency for all analyzed days of observations of spikes connected with active region No.260 are presented on the Fig.6.

We approximate these dependencies by the linear functions of kind $\Delta f \propto A \cdot f$. The coefficient of proportion $A$ equals 1.4, 2.4, 1.5, 2.5, 1.5, 1.6 for July 27 - August 2 correspondingly, i. e. it varies in the small range from 1.4 to 2.5 . As it will be shown further the spike bandwidth practically does not depend on day of observations or on active region position on the solar disk. This indicates that bandwidth does not depend on propagation effects of electromagnetic waves in the solar corona but determined only by conditions in the place of generation. Similar to solar S-bursts we assume that bandwidth of spikes determined by magnetic field. 


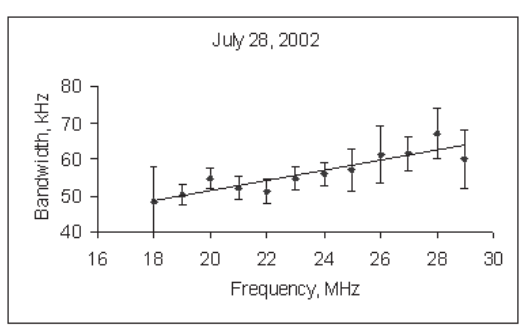

a)

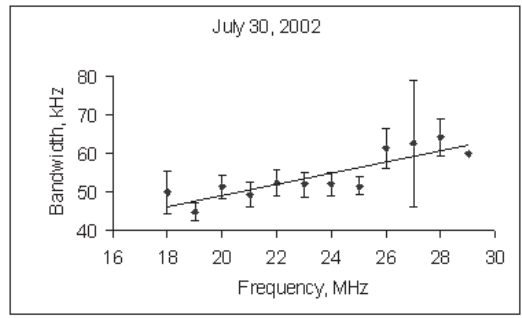

b)

Figure 6: The bandwidth dependence on frequency for July 28 (a) and July 30 (b), 2002.

\subsection{Fluxes of Decameter Spikes}

The decameter spikes do not comprise very large fluxes, but in most cases they are lower than 100 s.f.u. Flux dependencies on frequency are presented on Fig.7. From these dependencies follows that for the first four days the dependencies are rather steep with strong increase in the region of low frequencies and for the other three days the flux is practically unchanged.

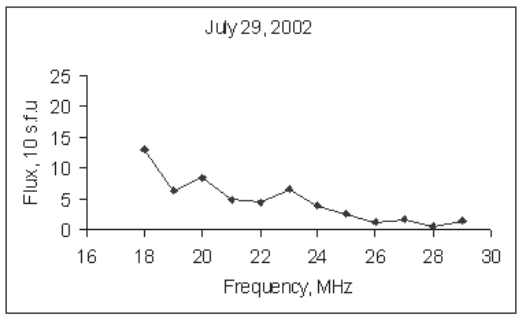

a)

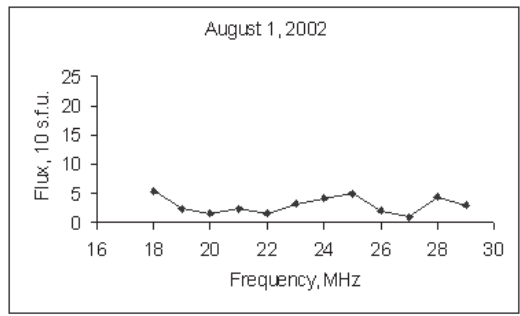

b)

Figure 7: Radio emission fluxes dependence on frequency for July 29 (a) and August 1(b), 2002.

\subsection{Dependence of Spike Parameters on Active Region Position}

The active region $N o .260$ associated with spikes activity appears on the east side of the solar limb on July 22 and disappears on the west side of it on August 3. During this period of time type III bursts storm simultaneously with spike bursts storm were observed. We consider variations of main parameters of spikes with change of locations of active region on the solar disk.

The example of variation of spikes duration on dependence from active region positions at frequency $20 \mathrm{MHz}$ is shown in Fig.8a, c, e. The minimum value of durations is observed on July 29 - 31 (Fig.8a, c, e) at frequencies 19 - $25 \mathrm{MHz}$. At the same time at frequencies 26 - $29 \mathrm{MHz}$ the duration remains more stable. In other words it does not depend from active region position on the solar disk. This fact is in agreement with dependence of 


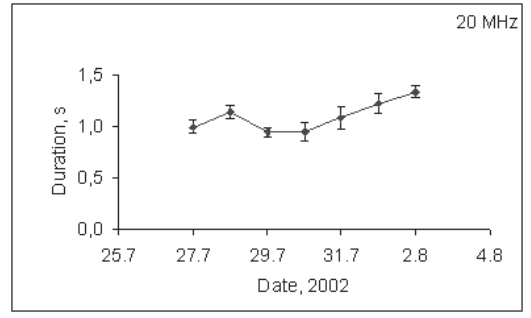

a)

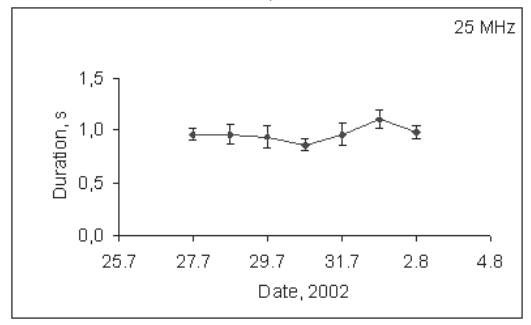

c)

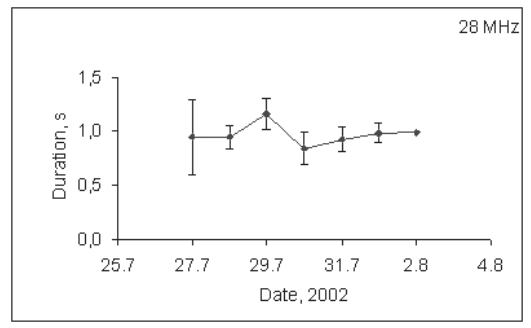

e)

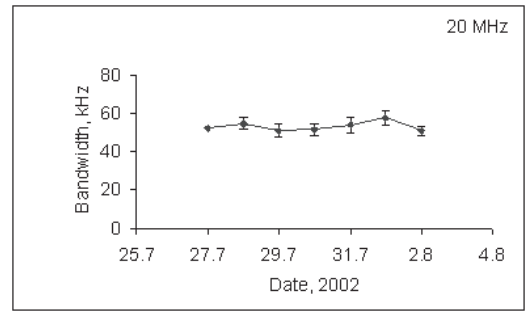

b)

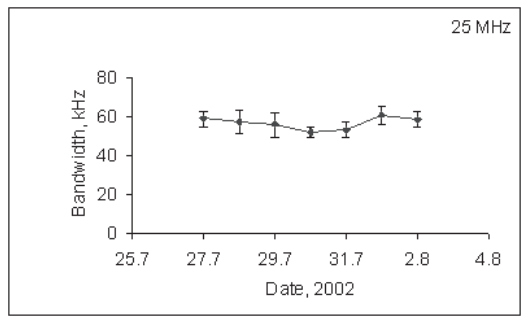

d)

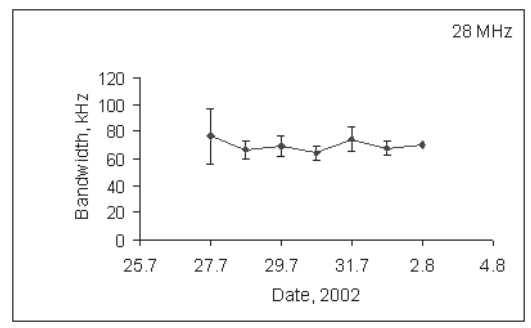

f)

Figure 8: Dependencies of spikes duration (a, c, e) and bandwidth (b, d, f) on active region position on the solar disk at frequencies $20 \mathrm{MHz}, 25 \mathrm{MHz}, 28 \mathrm{MHz}$.

spike duration on collision time of particles. The minimum of spikes duration observed on July 30 at all frequencies. In this day the active region was located in $20^{\circ}$ to the west from the central meridian. The frequency bandwidth of spikes is approximately constant for all days (Fig.8b, d, f). The property that frequency bandwidth does not depend from active region position indicates that it defines by the processes in the place of generation.

\section{Discussion}

We consider that spikes as type III bursts are generated by fast electron beams which propagate along open magnetic field lines. The main difference is that spikes are caused by beams of less densities and spatial sizes. The small density means that the quasilinear 
time $\tau_{q u} \approx\left(\omega_{p e} n^{\prime} / n\right)^{-1}\left(\omega_{p e}=\sqrt{4 \pi e^{2} n / m_{e}}\right.$ is the plasma frequency, $n^{\prime}$ is the beam density, $n$ is the density of the ambient plasma) is large and generation of Langmuir waves and electromagnetic waves begins high in the corona. Comparing the qausilinear time and the time of free electron propagation $t=R / V_{0}$ ( $R$ is the distance, $V_{0}$ is the beam velocity) we derive that electron beams with density $n^{\prime} \leq 1 \mathrm{~cm}^{-3}$ and velocity $V_{0}=10^{10} \mathrm{~cm} / \mathrm{s}$ start to radiate at frequencies $10-30 \mathrm{MHz}$. If the initial distribution function of electrons is stable then according to [Melnik et al., 2000] generation of Langmuir waves happens in the limited regions with sizes $d=\sqrt{2} l$, where $l$ is the spatial size of electron beam. We consider that namely from these regions the radio emission of spikes comes out. If the spatial sizes of electron beams are smaller than $\tau_{\text {coll }} v_{0} \approx 10^{10} \mathrm{~cm}$ durations of radiation from these regions are defined by particle collisions in the coronal plasma and this explains observed spike durations. Fast electron beams move along open magnetic field lines into some solid angle $\theta$. Electrons generate Langmuir waves $l$ near the high hybrid resonance frequency . These waves are transformed into electromagnetic waves $t$ at scattering on ions $i$ in the processes $l+i \rightarrow t+i$. Their frequency is approximately equal to $\omega_{p e}$ and the frequency bandwidth is defined by the equation $\Delta \omega=\omega_{B e}^{2} \sin ^{2} \theta / 2 \omega_{p e}\left(\omega_{B e}=e B / m c\right.$ is the electron cyclotron frequency). Comparison with empirical dependence $\Delta f \propto A \cdot f$ obtained above gives for $A$ equation $A=\omega_{B e}^{2} \sin ^{2} \theta / 2 \omega_{p e}^{2}$. Using the value of magnetic field $B=2 G$ found from data for S-bursts [Melnik, 2010] we derived the angle $\theta$ into which fast electrons were confined. It changes from $13 \mathrm{deg}$ (28 July) up to $18 \mathrm{deg}$ (29 and 31 July). Such values $\theta$ are agreed with estimates by Zaitsev [1974].

\section{Conclusion}

We consider that decameter spikes are low frequency extension of high frequencies spikes. Similar to them decameter spikes are observed simultaneously with different types of solar bursts and they have small duration. Such small durations of meter and decameter spikes can be explained by collision time of particles in plasma with temperature $10^{6} \mathrm{~K}$. Bandwidths of decameter spikes equal to $50-70 \mathrm{kHz}$ that is only a fraction $0.2-0.3 \%$ of the observational frequency. Meter and decameter spikes can be explained according to plasma mechanism of radiation. Decameter spikes are generated by fast electron beams similar to beams which responsible for type III bursts. But beams associated with spikes have smaller spatial sizes and smaller densities. Small density of the beams leads to generation of Langmure waves high in the solar corona near $1-2 R_{s}$ and consequently to appearance of spikes at frequencies of the decameter range. The bandwidth is determined by frequency bandwidth in which the Langmure waves were generated, magnetic field and angle in which fast electrons are propagate.

\section{References}

Benz, A. O., Millisecond radio spikes, Solar Phys., 104, 99-110, doi:0.1007/BF00159950, 1986. 
Bazelyan, L. L., N. Yu. Goncharov, V. V. Zaitsev, V. A. Zinichev, V. O. Rapoport, Ya. G. Tsybko, Frequency and time splitting of decameter solar radio bursts, Solar Phys., 39, 213-222, doi:10.1007/BF00154982, 1974.

De Groot, T. Inf. Bulletin Solar Radio Obs., 9, 4, 1962.

Malville, J. M., H. D. Aller, and C. J. Jensen, Spike bursts during the Type IV event of February, Amer. Astron. Soc., 711-717, 1967.

Melnik, V.N., E.P. Kontar, and V.I. Lapshin, Propagation of a Maxwellian Electron Cloud in a Plasma, Solar Phys., 196, 199-212, 2000.

Melnik, V. N., A. A. Konovalenko, H. O. Rucker, V. V. Dorovskyy, E. P. Abranin, A. Lecacheux, A. S. Lonskaya, Solar S-bursts at frequencies 10-30MHz, Solar Phys., 264, 103-117, 2010.

Melnik, V.N., A.A. Konovalenko, H.O. Rucker, V.V. Dorovskyy, E.P. Abranin, A. Lecacheux, and A.S. Lonskaya, Solar S-bursts at frequencies 10-30MHz, Solar Phys., 264, 103-117, 2010.

Zaitsev, V.V., Quasi-one-dimensional nature of the spectrum of plasma waves in type III radioburst sources, Astron. Zhur., 51, 801-812, 1974.

Zheleznyakov, V.V., and B.V. Zaitsev, Some features of solar spike burst generation, Astron. Astrophys., 39, 107-111, 1975. 